\title{
SDSS-IV MaNGA: 3D spin alignment of spiral and S0 galaxies
}

\author{
Katarina Kraljic ${ }^{1,2 \star}$, Christopher Duckworth ${ }^{3}$, Rita Tojeiro ${ }^{3}$, Shadab Alam ${ }^{2}$, \\ ${ }^{1}$ Institute for Astronomy, University of Edinburgh, Royal Observatory, Blackford Hill, Edinburgh, EH9 3HJ, United Kingdom \\ ${ }^{2}$ Aix Marseille Université, CNRS, CNES, UMR 7326, Laboratoire d'Astrophysique de Marseille, Marseille, France \\ ${ }^{3}$ School of Physics and Astronomy, University of St Andrews, North Haugh, St Andrews, KY16 9SS, United Kingdom \\ ${ }^{4}$ Apache Point Observatory and New Mexico State University, P.O. Box 59, Sunspot, NM, 88349-0059, USA \\ ${ }^{5}$ Sternberg Astronomical Institute, Moscow State University, Moscow, Russia \\ ${ }^{6}$ Department of Physics \& Astronomy, University of Utah, Salt Lake City, UT, 84112, USA \\ ${ }^{7}$ Instituto de Astronomía y Ciencias Planetarias de Atacama, Universidad de Atacama, Copayapu 485, Copiapó, Chile \\ ${ }^{8}$ Centro de Investigación en Astronomía, Universidad Bernardo O'Higgins, Avenida Viel 1497, Santiago, Chile
} Dmitry Bizyaev ${ }^{4,5}$, Anne-Marie Weijmans ${ }^{3}$, Nicholas Fraser Boardman ${ }^{6}$, Richard R. Lane $\mathrm{C}^{7,8}$

Accepted XXX. Received YYY; in original form ZZZ

\begin{abstract}
We investigate the 3D spin alignment of galaxies with respect to the large-scale filaments using the MaNGA survey. The cosmic web is reconstructed from the Sloan Digital Sky Survey using DiSPERSE and the 3D spins of MaNGA galaxies are estimated using the thin disk approximation with integral field spectroscopy kinematics. Late-type spiral galaxies are found to have their spins parallel to the closest filament's axis. The alignment signal is found to be dominated by low-mass spirals. Spins of S0-type galaxies tend to be oriented preferentially in perpendicular direction with respect to the filament's axis. This orthogonal orientation is found to be dominated by S0s that show a notable misalignment between their kinematic components of stellar and ionised gas velocity fields and/or by low mass S0s with lower rotation support compared to their high mass counterparts. Qualitatively similar results are obtained when splitting galaxies based on the degree of ordered stellar rotation, such that galaxies with high spin magnitude have their spin aligned, and those with low spin magnitude in perpendicular direction to the filaments. In the context of conditional tidal torque theory, these findings suggest that galaxies' spins retain memory of their larger-scale environment. In agreement with measurements from hydrodynamical cosmological simulations, the measured signal at low redshift is weak, yet statistically significant. The dependence of the spin-filament orientation of galaxies on their stellar mass, morphology and kinematics highlights the importance of sample selection to detect the signal.
\end{abstract}

Key words: galaxies: kinematics and dynamics, evolution, formation - cosmology: large scale Structures of the universe

\section{INTRODUCTION}

Angular momentum of galaxies is one of the key ingredients to understand their morphological diversity. As galaxies are on large scales organised within the network of filaments and walls, it is expected that this large-scale anisotropic environment is, at least partially, driving their morphology. Indeed, revisiting the tidal torque theory (TTT; Hoyle 1949; Peebles 1969; Doroshkevich 1970; White 1984; Catelan \& Theuns 1996; Lee \& Pen 2000, 2001, see also Schäfer 2009, for a review) in the context of such anisotropic environment, Codis et al. (2015) explained the relative angular momentum distribution of halos with respect to the filaments and walls of the cosmic web. The misalignment between the tidal and the inertia tensors constrained to the vicinity of filament-type saddle points implies a spin aligned with filaments for low mass halos,

^ E-mail: katarina.kraljic@lam.fr and a perpendicular spin orientation for more massive halos, in agreement with findings from cosmological N-body simulations (e.g. Aragón-Calvo et al. 2007; Hahn et al. 2007; Codis et al. 2012; Trowland et al. 2013; Wang \& Kang 2017; Ganeshaiah Veena et al. 2018). Recently, it was found that this transition mass (the critical mass at which the transition of the halo spin orientation occurs) is sensitive to the total neutrino mass (Lee et al. 2020) and to the dark energy model (Lee \& Libeskind 2020). The intrinsic spinshear alignment is therefore potentially powerful complementary probe of massive neutrinos and dark energy.

Galaxies seem to retain a memory of their spin orientation with respect to the cosmic web filaments and walls, as suggested by the results from large-scale cosmological hydrodynamical simulations (Dubois et al. 2014; Codis et al. 2018; Wang et al. 2018; Ganeshaiah Veena et al. 2019; Kraljic et al. 2020b). The mass dependence of the spin alignment signal is however debated. While some works confirmed the existence of a galaxy spin transi- 


\section{K. Kraljic et al.}

tion from parallel to perpendicular with respect to the filament's direction (Dubois et al. 2014; Codis et al. 2018; Kraljic et al. 2020b), and analogously with respect to walls (Codis et al. 2018; Kraljic et al. 2020b), others (Ganeshaiah Veena et al. 2019; Krolewski et al. 2019) found preferential perpendicular orientation with respect to filaments at all masses with no sign of a spin transition. A possible interpretation of this lack of detection of a clear transition is the nature of the filaments, with galaxies in thinner filaments having their spins more likely perpendicular to the filament's axis, compared to galaxies of similar mass in thicker filaments (Ganeshaiah Veena et al. 2019). This can be in turn understood recalling the multi-scale nature of the problem and the conditional TTT (Codis et al. 2015) predicting larger transition mass for denser, thus thicker, filaments. Further support for this interpretation was provided by the findings of stronger impact of large scale tides on the galaxy spin orientation in denser filaments (Kraljic et al. 2020b, using filament density as a proxy for the thickness of filaments). In addition to the stellar mass, the spin-filament alignment was shown to depend on other internal properties of galaxies. Blue or rotation-supported galaxies were found to dominate the alignment signal at low stellar mass, while red or dispersion-dominated galaxies tend to show a preferential perpendicular alignment (Codis et al. 2018; Wang et al. 2018; Kraljic et al. 2020b).

The orientation of the galaxies' spin with respect to their largescale environment has been quite extensively studied also on the observational side. When focusing on disc galaxies, some groups find preferentially parallel orientation for spirals (Tempel et al. 2013; Tempel \& Libeskind 2013), Scd types (Hirv et al. 2017), or both red and blue galaxies (Zhang et al. 2013). Others report a tendency for a perpendicular orientation for spirals (Lee \& Erdogdu 2007; Jones et al. 2010; Zhang et al. 2015) and Sab galaxies (Hirv et al. 2017), or no evidence for a clear signal (Pahwa et al. 2016; Krolewski et al. 2019). A much better agreement seems to exist for elliptical/S0 galaxies, for which a preferential orthogonal orientation of their spin (or minor axis) with respect to their host filaments is found (Tempel et al. 2013; Pahwa et al. 2016), in agreement with with results of shape measurements (e.g. Okumura \& Jing 2009; Joachimi et al. 2011; Singh et al. 2015; Chen et al. 2019; Johnston et al. 2019).

More recently, Welker et al. (2020) studied the alignment of galactic spin in projection with respect to the filaments of the cosmic web using the integral field spectroscopic (IFS) survey SAMI. They found a mass-dependence of the signal with low-mass galaxies aligning their spin with their nearest filament while their higher mass counterparts were found to more likely display an orthogonal orientation. Similarly, Blue Bird et al. (2020) found a preferential alignment of projected galaxy spins (using the photometric major axis of the stellar disc) with the cosmic filaments when analysing a sample of $\mathrm{H}$ I biased late-type low mass galaxies in the COSMOS H I Large Extragalactic Survey (CHILES) sample. On the other hand, Krolewski etal. (2019) found no evidence for alignment in projection between galaxy spins, measured from Mapping Nearby Galaxies at Apache Point Observatory (MaNGA) kinematics and filaments from the SDSS Main Galaxy Sample. There is currently no clear consensus on these various observations. However, these differences are not surprising, given that the signal is expected to be weak and yarious selection criteria need to be carefully examined.

In this work, we measure the alignment between filaments identified in the SDSS Main Galaxy Sample and galaxy spins measured from MaNGA kinematics in 3D. This is the first measurement of the spin alignment in 3D with the use of IFU spectroscopy kinematics. The outline of this paper is as follows. In Section 2, we briefly describe the data and methods used in this work. Section 3 presents the results on the alignment of spiral and S0-type galaxies. In Section 4, we summarise and discuss our findings.

\section{DATA AND METHODS}

\subsection{The MaNGA survey}

The MaNGA survey (Mapping Galaxies at Apache Point; Bundy et al. 2015; Wake et al. 2017), one of three programs in the fourth generation of the Sloan Digital Sky Survey (SDSS-IV; Blanton et al. 2017), is designed to investigate the internal structure of $\sim 10000$ galaxies in the nearby Universe $(0<z<0.15)$. Detailed kinematics are enabled by integral field unit (IFU) spectroscopy, which uses the 2.5-metre telescope at the Apache Point Obseryatory (Gunn et al. 2006) along with the two channel BOSS spectrographs (Smee et al. 2013) and the MaNGA IFUs (Drory et al. 2015). MaNGA provides spatial resolution on kpc scales (2" diameter fibres) while covering $3600-10300 \AA$ in wavelength with a spectral resolution of $\mathrm{R} \sim 2000$.

MaNGA observations are covered plate by plate, employing a dithered pattern for each galaxy corresponding to one of the 17 fibre-bundles of 5 distinct sizes. Any incomplete data release of MaNGA should therefore be unbiased with respect to IFU sizes and hence a reasonable representation of the final sample scheduled to be complete in 2020. The sizes of the IFU are matched to each galaxy in the Primary sample to give a minimum coverage of 1.5 effective radii $\left(R_{e}\right)$ (Law et al. 2015). The Secondary sample covers up-to a minimum of $2.5 R_{e}$ and the Colour-Enhanced supplement up-to $1.5 R_{e}$. All together these three sub-samples produce a galaxy population that is unbiased towards morphology, inclination and colour and provides a near flat distribution in stellar mass, $M_{*}$ A full description of the MaNGA observing strategy is given in Law et al. (2015); Yan et al. (2016b).

The raw data cubes are calibrated (Yan et al. 2016a) and reduced by the Data Reduction Pipeline (DRP; Law et al. 2016). The fibre flux and inverse variance is extracted from each exposure, which are then wavelength calibrated, flat-fielded and sky subtracted. In this work we use 4633 unique galaxies from the 7th data release of MaNGA (known internally as MaNGA Product Launch 7), which corresponds to data released as part of SDSS-IV data release 15 (Aguado et al. 2019).

\subsection{Stellar kinematics}

For a complete description of the estimation of stellar kinematics, we direct the reader to the MaNGA Data Analysis Pipeline (DAP; Westfall et al. 2019) from which our data are taken from. We summarise the key points here. Stellar kinematics are extracted using the Penalised Pixel-Fitting method (Cappellari \& Emsellem 2004; Cappellari 2017), which fits the stellar continuum on a spaxel (spectral pixel) by spaxel basis. The line of sight of velocity dispersion is extracted and then absorption-line spectra are fitted using a set 49 clusters of stellar spectra from the MILES stellar library (Sánchez-Blázquez et al. 2006; Falcón-Barroso et al. 2011). We use spectra that are spatially Voronoi binned to $g$-band $S / N$ 10 while excluding any individual spectrum with a $g$-band $S / N<1$ (Cappellari \& Copin 2003).

We estimate the global position angle (PA) of the stellar velocity fields using the fit_kinematic_pa routine (see Appendix 
C of Krajnović et al. 2006). We remove background galaxies within the IFU and define PAs in the interval $0-180^{\circ}$ (east of north). To select a clean sample only galaxies with well defined global PAs are considered (see Duckworth et al. 2019, 2020, for more details). Note that such a requirement naturally excludes gas-poor and slowly rotating elliptical galaxies (the majority of removed galaxies have largely incomplete velocity fields, see Duckworth et al. 2019, for more details). However, as the focus of this work is on late-type and lenticular galaxies (as described below), this does not impact our results.

\subsection{NSA catalogue}

The MaNGA survey is targeted from an adapted version of the NASA Sloan Atlas (NSA V1_0_1 ${ }^{1}$ ) which is based on SDSS imaging (Blanton et al. 2011). For each galaxy selected from DR15, we take the stellar mass found using a K-correction (Blanton \& Roweis 2007), the axial ratio (b/a) and photometric position angle from the parent NSA catalogue. These parameters are derived using a set of elliptical annuli (based on the Petrosian radius), which maintain the stability of circularised fits to photometry but without the systematic biases of two-component Sérsic fits (Wake et al. 2017).

\subsection{Morphology}

Owing to the method used to compute the $3 \mathrm{D}$ angular momentum of galaxies, we focus in this work on galaxies possessing a disk component, namely late-type (LTGs) and lenticular (S0s) galaxies.

To classify galaxies into LTGs, we use morphological classifications taken from the second iteration of the citizen science project; GalaxyZoo (GZ; Willett et al. 2013). GZ relies on visual inspection from volunteers of the general public, who answer a series of questions to characterise morphological type and identify finer features of each galaxy (see Figure 1 in Willett et al. 2013, for specific questions). The final catalogue provides a set of weighted responses from all volunteers which are averaged to provide a probability for each classification (e.g. 'Is the galaxy smooth and rounded with no sign of a disk?'). Classifications in MaNGA are taken from a combination of GZ2 and GZ4 data releases ${ }^{2}$.

Identification of S0s relies on morphological classifications based on the the Deep Learning (DL) scheme introduced in Domínguez Sánchez et al. (2018); here we give a quick outline. The algorithm is trained on two visually-based morphological catalogues; citizen science project GZ2 and expert classifications of Nair \& Abraham (2010). Morphology is defined for each MaNGA galaxy using SDSS photometry. The DL algorithm estimates a morphological T-type (e.g. see; Nair \& Abraham 2010, for more information) which is first used to separate each galaxy into ETGs and LTGs. To select lenticulars, all ETGs are assigned a probability of being S0 $\left(P_{S 0}\right)$ based on the presence of disk structure and dominance of the bulge. All DL classifications are finally eye-balled to check for reliability.

The choice of the DL classification for the construction of the sample of lenticulars instead of GZ is motivated by a need of a clean sample of S0s. GZ classification allows for classification of galaxies into a S0-Sa type (from an empirical formula transforming vote fractions; Equation 19 in Willett et al. 2013), which is not optimal

1 www.sdss.org/dr15/manga

2 manga-morphologies-from-galaxy-zoo for the purposes of this study. Visual inspection of galaxies falling in this category confirmed the presence of a large fraction of galaxies with prominent spiral arms, that are absent from the sample of S0s based on the DL scheme.

On the other hand, GZ classification allows for a selection of highly probable LTGs. Such a clean sample of LTGs is crucial for a detection of a spin-filament alignment signal that is expected to be weak.

\subsection{The Cosmic web}

The filaments of the cosmic web are extracted using the publicly available code DISPERSE (Sousbie 2011; Sousbie et al. 2011) ${ }^{3}$, which identifies cosmic web structures with a parameter- and scalefree topologically motivated algorithm. For the purposes of this work, DISPERSE was run with a $3 \sigma$ persistence threshold on the Tempel et al. (2014) SDSS galaxy catalogue (for more details, see e.g. Duckworth et al. 2019; Kraljic et al. 2020a).

\subsection{Angular momentum}

To compute the spin of galaxies, we adopt the thin-disk approximation following Lee \& Erdogdu (2007). Here we summarise the main steps.

The spin axis of a galaxy in the local spherical coordinate system can be written as

$\hat{L}_{r}=\cos i$,

$\hat{L}_{\theta}=\left(1-\cos ^{2} i\right)^{1 / 2} \sin \mathrm{PA}$,

$\hat{L}_{\phi}=\left(1-\cos ^{2} i\right)^{1 / 2} \cos \mathrm{PA}$,

where PA is the position angle and $i$ is the inclination angle of a galaxy. If not stated differently, the position angle PA used throughout this work refers to the kinematic PA as described in Section 2.2. The inclination angle $i$ can be computed, following Haynes \& Giovanelli (1984), as

$\cos ^{2} i=\frac{(b / a)^{2}-p^{2}}{1-p^{2}}$

where $b / a$ is the axis ratio and $p$ the intrinsic flatness parameter that varies with galaxy morphology (Haynes \& Giovanelli 1984). This parameter accounts for the fact that in practice, the disk of galaxies has finite thickness and the presence of a bulge would have an impact on the $b / a$. The proposed values for $p$ span range from 0.23 for S0 to 0.1 for Scd-Sdm types. We adopted the mean value of 0.158 , but choosing extreme values does not change our results. The value of $i$ is set to $\pi / 2$ if $b / a<p$.

The equatorial Cartesian coordinates of the unit spin vector can then be written as

$\hat{L}_{x}=\hat{L}_{r} \sin \alpha \cos \beta+\hat{L}_{\theta} \cos \alpha \cos \beta-\hat{L}_{\phi} \sin \beta$

$\hat{L}_{y}=\hat{L}_{r} \sin \alpha \sin \beta+\hat{L}_{\theta} \cos \alpha \sin \beta+\hat{L}_{\phi} \cos \beta$

$\hat{L}_{z}=\hat{L}_{r} \cos \alpha-\hat{L}_{\theta} \sin \alpha$,

where $\alpha=\pi / 2-$ DEC and $\beta=$ RA, with DEC and RA corresponding to declination and right ascension, respectively. We apply positive sign to $\hat{L}_{r}$ to all galaxies. Note that this sign ambiguity is expected to decrease the strength of the alignment signal. Alternatively, one can take into account the two-fold ambiguity statistically

\footnotetext{
3 http://www2.iap.fr/users/sousbie/web/html/indexd41d.html
} 
by considering both the clock wise and counter-clock wise rotations (Lee 2011). Such an approach does not alter the conclusions of this paper (see Appendix A).

\section{SPIN ALIGNMENT}

To measure the spin alignment, each galaxy is assigned its closest filament's segment, defined by a pair of points providing the local direction of the filament for a given galaxy. The absolute value of cosine of the angle between the spin of the galaxy and its closest filament, $|\cos \gamma|$ is used to assess the alignment with respect to the filamentary network of the cosmic web. Values of $|\cos \gamma|$ close to 1 indicate that galaxy tends to have its spin aligned with the neighbouring filament, while values close to 0 mean that the spin is in the orthogonal direction to the filament's axis. In order to increase the statistics of the measured signal, each galaxy is assigned two closest filaments' segments. In addition, we consider only galaxies that are not too faraway from the filaments $(\leq 13 \mathrm{Mpc})$ and not too close to the nodes of the cosmic web $(\geq 0.5 \mathrm{Mpc})^{4}$. To quantify the likelihood whether the measured alignments are consistent with being derived from a random distribution, a Kolmogorov-Smirnov (KS) test was performed on each distribution. To account for any possible observational bias, instead of comparing to a uniform distribution, we construct 2000 realisations of random samples by shuffling galaxy positions, i.e. by randomising the association between galaxies and their closest filaments. For indicative purposes, we show also statistical errors computed by bootstrapping considering 100 random samples drawn from the parent sample with replacement.

\subsection{Spirals}

To construct the sample of spiral or late-type galaxies (LTGs), we consider only galaxies with a fraction of debiased classification votes of 0.9 , somewhat more conservatively than in Duckworth et al. (2020). This choice is motivated by our desire to construct a clean sample of LTGs while keeping a reasonable number of galaxies.

Figure 1 shows the probability distribution function (PDF) of the cosine of the angle between the spin of galaxies and the direction of their closest filament $|\cos \gamma|$ for the entire sample of LTGs (top) and for low-mass $\left(M_{\star} \leq 10{ }^{10} \mathrm{M}_{\odot}\right)$ LTGs (bottom). Spiral galaxies tend to have their spin aligned with the neighbouring filaments. The alignment signal is found to be dominated by low mass spirals. We measure the spin of the galaxies from the stellar component (solid lines), however similar results are obtained when considering galaxies with a low offset between the global position angles of stellar and $\mathrm{H} \alpha$ velocity fields, $\triangle \mathrm{PA}<10^{\circ}$ (dotted line). As shown in Duckworth et al. (2019, 2020), only a small fraction of LTGs in the MaNGA sample show a significant misalignment. Interestingly, when using the photometric position angle from the NSA catalogue to compute the spin of galaxies, the measured signal is still statistically significant $\left(p_{\mathrm{KS}}<0.05\right)$, but much weaker (see Table 1$)^{5}$.

4 The values of these distances cuts are a result of a compromise between a clean and/sufficiently large sample. As expected, including galaxies that are at yery large distances from the filamentary network or that are in the highest density regions, typically galaxy groups and clusters, decreases the strength of the signal.

5 Note that the number of galaxies with the kinematic PA is lower com-

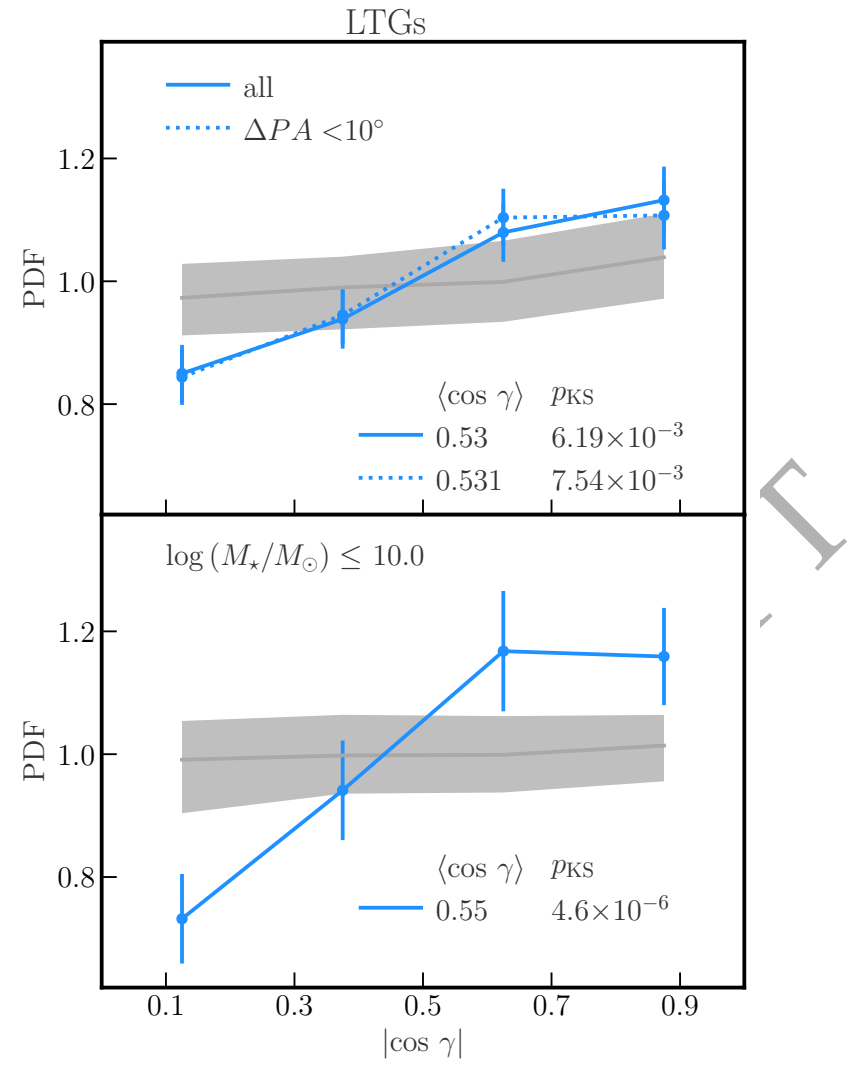

Figure 1. Alignment between the filaments and spin of all (top), and, lowmass (bottom) LTGs. The alignment signal for the kinematically aligned (i.e. $\triangle \mathrm{PA}<10^{\circ}$ ) galaxies only is shown by the dotted line. The error bars correspond to the bootstrap. The solid grey line and shaded area represent the median and 95 per cent confidence limits from 2000 random samples, respectively. The mean alignment angle together with the probability $p_{\mathrm{KS}}$ of the KS test are shown in each panel with corresponding symbols. LTGs in the probed mass range tend to have their spin parallel to their host filaments. The alignment signal seen for the entire population of LTGs is driven by the low mass sub-sample $\left(M_{\star} \leq 10^{10} \mathrm{M}_{\odot}\right)$.

\subsection{S0 galaxies}

To select S0 galaxies we consider all ETGs (T-type $\leq 0$ ) with $P_{S 0}>0.5$, in-keeping with Domínguez Sánchez et al. (2018). The selection criterion for S0 galaxies requiring more than 50 per cent of debiased classification votes is somewhat less conservative compared to the selection of LTGs (and also compared to e.g. Duckworth et al. 2019), and motivated by low number statistics of the sample. 6

Figure 2 shows the PDF of the cosine of the angle between the spin of galaxies and the direction of their closest filament $|\cos \gamma|$ for the entire sample of S0s (top) and for low-mass $\left(M_{\star} \leq 10^{10} \mathrm{M}_{\odot}\right)$ S0s (bottom). The spin of S0 galaxies is found to be preferentially orthogonal to the the neighbouring filaments. As for LTGs, the low-mass sub-sample of S0s seem to dominate the signal. On the other hand, the preferential orthogonal orientation of the spin is found to be driven by kinematically misaligned galaxies, defined as

pared to the photometric measurement due to the additional quality cuts (see Section 2.2). This does not alter our conclusion.

6 We note however that restricting this condition to over 70 per cent yields qualitatively similar results, albeit with reduced statistical significance. 


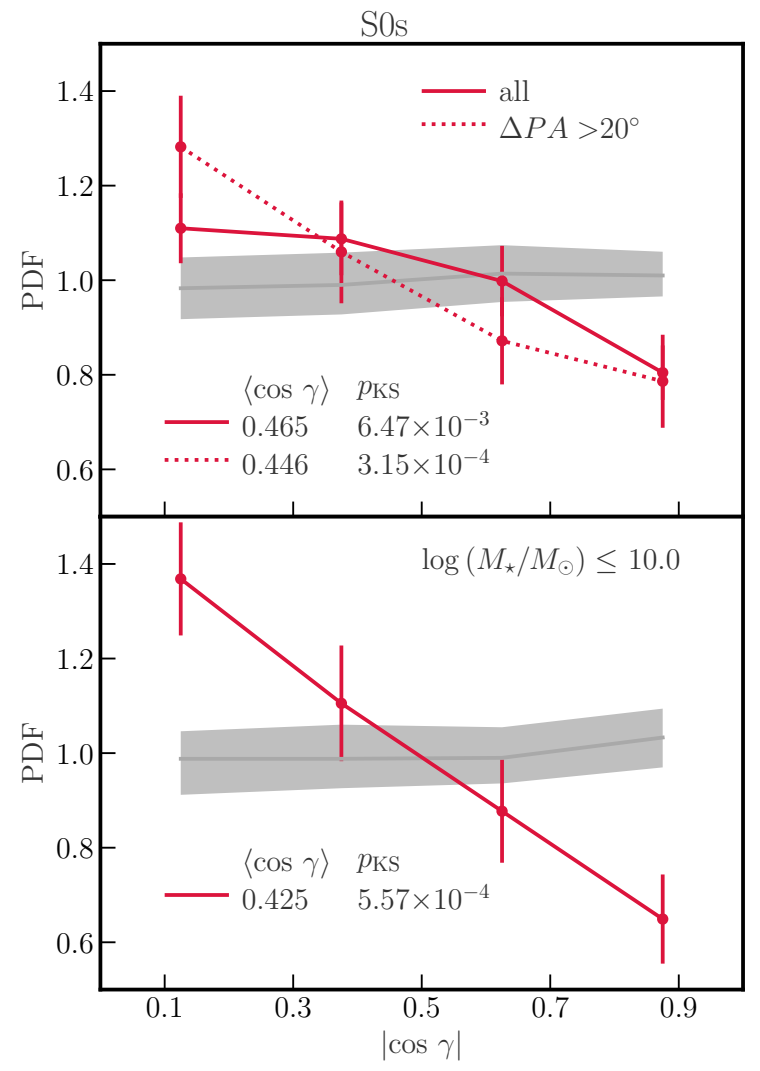

Figure 2. Alignment between the filaments and spin of all (top) and lowmass (bottom) S0s. The error bars correspond to the bootstrap. The solid grey line and shaded area represent the median and 95 per cent confidence limits from 2000 random samples, respectively. The mean alignment angle together with the probability $p_{\mathrm{KS}}$ of the KS test are shown in each panel with corresponding symbols. S0s in the probed mass range tend to have their spin perpendicular to their host filaments. The alignment signal seen for the entire population of S0s is driven by the kinematically misaligned $\left(\triangle P A>20^{\circ}\right.$; red dotted line on the top panel) and low mass sub-sample $\left(M_{\star} \leq 10^{10} \mathrm{M}_{\odot}\right)$.

$\triangle \mathrm{PA}>20^{\circ}$ (dotted line). As in the case of LTGs, when using the photometric position angle from the NSA catalogue to compute the spin of galaxies, the measured signal is still statistically significant $\left(p_{\mathrm{KS}}<0.05\right)$, but much weaker (see Table 1$)$.

\section{DISCUSSION AND CONCLUSIONS}

In this work, we present the first measurement of the alignment of galaxy spins with respect to their cosmic filaments with the use of IFS kinematics in 3D. Galaxy spin is reconstructed using the thindisk approximation applied to galaxies from the MaNGA survey. The filamentary network is reconstructed in 3D from the galaxy distribution in the SDSS survey. Our main results are as follows.

- LTGs/spiral galaxies tend to have their spin aligned with the axis of their neighbouring filament.

- The alignment signal for LTGs is dominated by low-mass galaxies, with $M_{\star} \leq 10^{10} \mathrm{M}_{\odot}$.

- S0-type galaxies have their spin preferentially in orthogonal direction with respect to their closest filament.

- The perpendicular orientation of S0s' spins is dominated by low-
Table 1. Number of galaxies, average $|\cos \gamma|$ and the KS probability $p_{\mathrm{KS}}$ that the sample is drawn from a random distribution for various sub-samples of LTGs, S0s, and galaxies with $\lambda_{R}>0.73$ and $\lambda_{R}<$ 0.4 . For LTGs and S0s, we provide also the results for photometric data (shown in parenthesis).

\begin{tabular}{|c|c|c|c|c|}
\hline & selection & $N_{\mathrm{gal}}$ & $\langle\cos \gamma\rangle$ & $p_{\mathrm{KS}}$ \\
\hline \multirow{4}{*}{ LTGs } & all & $\begin{array}{c}611 \\
(852)\end{array}$ & $\begin{array}{c}0.53 \\
(0.515)\end{array}$ & $\begin{array}{c}6.2 \times 10^{-3} \\
\left(2.3 \times 10^{-2}\right)\end{array}$ \\
\hline & $M_{\star} \leq 10^{10} \mathrm{M}_{\odot}$ & $\begin{array}{c}230 \\
(377)\end{array}$ & $\begin{array}{c}0.55 \\
(0.521)\end{array}$ & $\begin{array}{c}4.6 \times 10^{-6} \\
\left(1.9 \times 10^{-2}\right)\end{array}$ \\
\hline & $M_{\star}>10^{10} \mathrm{M}_{\odot}$ & $\begin{array}{c}381 \\
(475)\end{array}$ & $\begin{array}{l}0.517 \\
(0.5)\end{array}$ & $\begin{array}{c}6.4 \times 10^{-1} \\
\left(4.8 \times 10^{-1}\right)\end{array}$ \\
\hline & $\Delta \mathrm{PA}<10^{\circ}$ & 556 & 0.53 & $7.5 \times 10^{-3}$ \\
\hline \multirow{4}{*}{ SOs } & all & $\begin{array}{c}269 \\
(363)\end{array}$ & $\begin{array}{c}0.465 \\
(0.482)\end{array}$ & $\begin{array}{c}6.5 \times 10^{-3} \\
\left(4.5 \times 10^{-2}\right)\end{array}$ \\
\hline & $M_{\star} \leq 10^{10} \mathrm{M}_{\odot}$ & $\begin{array}{c}114 \\
(170)\end{array}$ & $\begin{array}{c}0.425 \\
(0.447)\end{array}$ & $\begin{array}{l}\left.5.6 \times 10^{-4}\right) \\
\left(2.9 \times 10^{-3}\right)\end{array}$ \\
\hline & $M_{\star}>10^{10} \mathrm{M}_{\odot}$ & $\begin{array}{c}155 \\
(193)\end{array}$ & $\begin{array}{c}0.496 \\
(0.513)\end{array}$ & $\begin{array}{l}\left.5.9 \times 10^{-1}\right) \\
\left(5.9 \times 10^{-1}\right)\end{array}$ \\
\hline & $\Delta \mathrm{PA}>20^{\circ}$ & 117 & 0.446 & $3.2 \times 10^{-4}$ \\
\hline \multirow[b]{2}{*}{$\lambda_{\mathrm{R}}>0.73$} & all & 131 & 0.554 & $9.0 \times 10^{-3}$ \\
\hline & $\begin{array}{c}M_{\star} \leq 10^{10} \mathrm{M}_{\odot} \\
M_{\star}>10^{10} \mathrm{M}_{\odot} \\
\Delta \mathrm{PA}<10^{\circ}\end{array}$ & $\begin{array}{c}71 \\
60 \\
130\end{array}$ & $\begin{array}{l}0.593 \\
0.508 \\
0.557\end{array}$ & $\begin{array}{l}5.4 \times 10^{-3} \\
7.5 \times 10^{-1} \\
1.0 \times 10^{-2}\end{array}$ \\
\hline$\lambda_{\mathrm{R}}<0.4$ & $\begin{array}{c}\text { all } \\
M_{\star} \leq 10^{10} \mathrm{M}_{\odot} \\
M_{\star}>10^{10} \mathrm{M}_{\odot} \\
\Delta \mathrm{PA}>20^{\circ}\end{array}$ & $\begin{array}{l}133 \\
211 \\
116\end{array}$ & $\begin{array}{l}0.479 \\
0.447 \\
0.499 \\
0.445\end{array}$ & $\begin{array}{l}3.0 \times 10^{-2} \\
6.5 \times 10^{-5} \\
3.4 \times 10^{-1} \\
2.2 \times 10^{-3}\end{array}$ \\
\hline
\end{tabular}

mass $\left(M_{\star} \leq 10^{10} \mathrm{M}_{\odot}\right)$ and/or kinematically misaligned galaxies, with $\triangle \mathrm{PA}>20^{\circ}$

- Qualitatively similar trends are recovered when using photometric data for the spin de-projection instead of IFS kinematics, albeit weaker and with lower statistical significance.

Our findings of the preferential spin-filament alignment for spiral galaxies and orthogonal orientation for S0s is in agreement with Tempel et al. (2013) studying the alignment of these two populations of galaxies using the SDSS Main Galaxy sample. These results are also broadly in agreement with the trends seen in hydrodynamical simulations (Codis et al. 2018; Kraljic et al. 2020b) showing that at fixed stellar mass the alignment signal is dominated by galaxies with high $v / \sigma$ (ratio of rotation to dispersion dominated velocities, used as a proxy for morphology), while the perpendicular orientation is mostly dominated by galaxies with low values of $v / \sigma$.

A decreasing strength of the alignment signal for MaNGA spirals with increasing stellar mass is also in a good agreement with these simulations (and reported stellar mass dependent projected spin flip for SAMI galaxies, Welker et al. 2020). Our results for S0type galaxies appear to be at odds with the predicted stellar mass dependence of the signal. One would naively expect that massive S0s should dominate the perpendicular spin-filament orientation signal, while the opposite is found. We note however, that a direct comparison with the simulations is not straightforward. This is because the thin-disk approximation used in this work to de-project the spin of galaxies cannot be applied to pure early-type galaxies that are found to dominate the orthogonal spin-filament orientation of massive simulated galaxies. S0-type galaxies are not all slow rotators, they occupy a broad range of the ellipticity $(\epsilon)$-stellar angular momentum estimator $\left(\lambda_{\mathrm{R}}\right.$; see e.g. Emsellem et al. 2007) parameter space. In addition, kinematically misaligned galaxies tend to reside closer to the slow rotator regime regardless their morphol- 

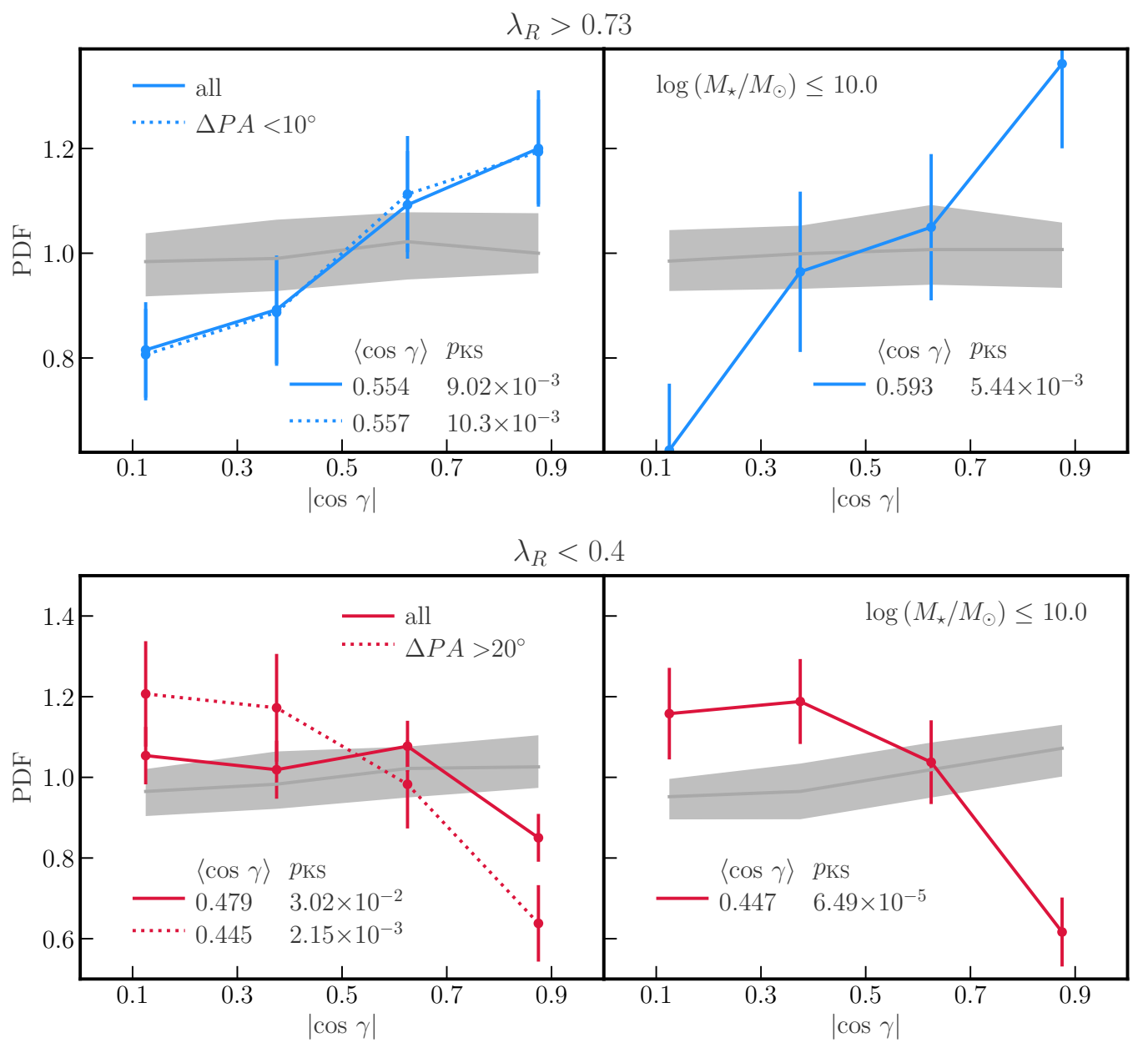

Figure 3. Top: Alignment between the filaments and spin of all (left) and low-mass (right) galaxies with high spin magnitude $\left(\lambda_{R}>0.73\right)$. Bottom: Alignment between the filaments and spin of all (left) and low-mass (right) galaxies with low spin magnitude $\left(\lambda_{R}<0.4\right)$. The error bars correspond to the bootstrap. The solid grey line and shaded area represent the median and 95 per cent confidence limits from 2000 random samples, respectively. The mean alignment angle together with the probability $p_{\mathrm{KS}}$ of the KS test are shown in each panel with corresponding symbols. Galaxies with high spin magnitude in the probed mass range tend to have their spin parallel to their host filaments. The alignment signal seen for the entire population is driven by the low mass sub-sample $\left(M_{\star}\right.$ $\leq 10^{10} \mathrm{M}_{\odot}$ ). Galaxies with low spin magnitude tend to have their spin oriented in perpendicular direction with respect to the filaments. The signal seen for the entire population is driven by the the kinematically misaligned $\left(\Delta P A>20^{\circ}\right.$; red dotted line on the left panel) and low mass sub-sample $\left(M_{\star} \leq 10^{10} \mathrm{M}_{\odot}\right)$.

ogy type (see Duckworth et al. 2020, their Fig. 8) and to populate lower stellar masses compared with aligned galaxies of the same morphology (Duckworth et al. 2019). This is consistent with our finding of low-mass and/or misaligned S0s dominating the orthogonal spin-filament orientation. We also find that on average, massive S0s tend to have higher values of $\lambda_{\mathrm{R}}$ (see Duckworth et al. 2020, and their Eq. 2 for details on the computation of the luminosity weighted $\lambda_{\mathrm{R}}$ from the IFU data) compared to their lower mass counterparts $(0.364 \pm 0.017$ and $0.323 \pm 0.011$, respectively $)$ and compared to kinematically misaligned counterparts $(0.24 \pm 0.01)$. A question that naturally arises is whether the parallel vs orthogonal orientation of spin of galaxies with respect to filaments of the cosmic web reflects the degree of ordered (stellar) rotation rather than their morphology. To this purpose, we combined all LTGs and S0s, and split the sample based on their $\lambda_{\mathrm{R}}$. The strongest alignment signal was obtained for galaxies with $\lambda_{\mathrm{R}}>0.73$ and orthogonal signal for galaxies with $\lambda_{\mathrm{R}}<0.4$. Galaxies with $\lambda_{\mathrm{R}}>0.73$ tend to be more aligned (higher $\langle\cos \gamma\rangle$ ) than the morphology-based sample of LTGs, however the statistical significance of the signal is somewhat lower ( $p_{\mathrm{KS}}$ values tend to be larger, see Table 1). On the other hand, galaxies with $\lambda_{\mathrm{R}}<0.4$ show weaker orthogonal signal (higher $\langle\cos \gamma\rangle$ ) compared to the morphology-based sample of S0s. While this suggests that morphology plays a role in determining the spin orientation with respect to the cosmic web filaments, it is hard at this stage to disentangle kinematics from morphology. Our analysis shows that splitting in either property gives different, but consistent cosmic web alignment signal, however we caution that much larger statistical sample is needed to study the dependence of the spin alignment at fixed stellar mass and morphology for slow and fast rotators.

This work underlines the importance of carefully constructing the sample of galaxies when assessing the spin-filament signal, given how it depends on stellar mass, morphological type and degree to which their rotation is dominated by dispersion motion. Together with the expectation that at low redshift the alignment is weak (or weaker compared to high redshift, see e.g. Codis et al. 2018), it could also explain the difficulty in detecting the signal in previous studies (e.g. Krolewski et al. 2019). It also highlights that the IFS kinematics allows to recover a stronger signal compared to photometric data alone. Altogether, this clearly motivates 
the need for larger IFS surveys such as e.g. the IFS Hector survey (Bland-Hawthorn et al. 2011; Bryant et al. 2016). These upcoming surveys will be spanning not only large range of stellar masses, but also large-scale environment, allowing to extend the existing studies on the spin-filament alignment using MaNGA (this work and that of Krolewski et al. 2019) and SAMI IFS surveys (Welker et al. 2020).

\section{DATA AVAILABILITY}

The data underlying this article are publicly available and will be shared on reasonable request to the corresponding author.

\section{ACKNOWLEDGEMENTS}

The authors thank the anonymous referee for comments that helped improve the clarity of the paper. We thank Stéphane Rouberol for the smooth running of the HORIZON Cluster, where some of the post-processing was carried out. We thank Thierry Sousbie for provision of the DISPERSE code (ascl.net/1302.015). KK thanks Christophe Pichon for his exceptional support, enthusiasm and exacting attention to details that have been a great source of inspiration. KK also warmly thanks Jounghun Lee for enlightening discussions and helpful feedback.

Funding for the Sloan Digital Sky Survey IV has been provided by the Alfred P. Sloan Foundation, the U.S. Department of Energy Office of Science, and the Participating Institutions.

SDSS-IV acknowledges support and resources from the Center for High Performance Computing at the University of Utah. The SDSS website is www.sdss.org.

SDSS-IV is managed by the Astrophysical Research Consortium for the Participating Institutions of the SDSS Collaboration including the Brazilian Participation Group, the Carnegie Institution for Science, Carnegie Mellon University, Center for Astrophysics I Harvard \& Smithsonian, the Chilean Participation Group, the French Participation Group, Instituto de Astrofísica de Canarias, The Johns Hopkins University, Kavli Institute for the Physics and Mathematics of the Universe (IPMU) / University of Tokyo, the Korean Participation Group, Lawrence Berkeley National Laboratory, Leibniz Institut für Astrophysik Potsdam (AIP), Max-PlanckInstitut für Astronomie (MPIA Heidelberg), Max-Planck-Institut für Astrophysik (MPA Garching), Max-Planck-Institut für Extraterrestrische Physik (MPE), National Astronomical Observatories of China, New Mexico State University, New York University, University of Notre Dame, Observatário Nacional / MCTI, The Ohio State University, Pennsylvania State University, Shanghai Astronomical Observatory, United Kingdom Participation Group, Universidad Nacional Autónøma de México, University of Arizona, University of Colorado Boulder, University of Oxford, University of Portsmouth, Uniyersity of Utah, University of Virginia, University of Washington, University of Wisconsin, Vanderbilt University, and Yale Uniyersity.

\section{REFERENCES}

Aguado D. S., et al., 2019, ApJS, 240, 23

Aragón-Calvo M. A., van de Weygaert R., Jones B. J. T., van der Hulst J. M., 2007, ApJ, 655, L5

Bland-Hawthorn J., et al., 2011, Optics Express, 19, 2649

Blanton M. R., Roweis S., 2007, AJ, 133, 734
Blanton M. R., Kazin E., Muna D., Weaver B. A., Price-Whelan A., 2011, AJ, 142, 31

Blanton M. R., et al., 2017, AJ, 154, 28

Blue Bird J., et al., 2020, MNRAS, 492, 153

Bryant J. J., et al., 2016, in Proc. SPIE. p. $99081 \mathrm{~F}$ (arXiv: 1608.03921), doi:10.1117/12.2230740

Bundy K., et al., 2015, ApJ, 798, 7

Cappellari M., 2017, MNRAS, 466, 798

Cappellari M., Copin Y., 2003, MNRAS, 342, 345

Cappellari M., Emsellem E., 2004, PASP, 116, 138

Catelan P., Theuns T., 1996, MNRAS, 282, 455

Chen Y.-C., Ho S., Blazek J., He S., Mandelbaum R., Melchior P., Singh S., 2019, MNRAS, 485, 2492

Codis S., Pichon C., Devriendt J., Slyz A., Pogosyan D., Dubois Y., Sousbie T., 2012, MNRAS, 427, 3320

Codis S., Pichon C., Pogosyan D., 2015, MNRAS, 452, 3369

Codis S., Jindal A., Chisari N. E., Vibert D., Dubois Y., Pichon C., Devriendt J., 2018, MNRAS, 481, 4753

Domínguez Sánchez H., Huertas-Company M., Bernardi M., Tuccillo D., Fischer J. L., 2018, MNRAS, 476, 3661

Doroshkevich A. G., 1970, Astrophysics, 6, 320

Drory N., et al., 2015, AJ, 149, 77

Dubois Y., et al., 2014, MNRAS, 444, 1453

Duckworth C., Tojeiro R., Kraljic K., Sgró M. A., Wild V., Weijmans A.-M., Lacerna I., Drory N., 2019, MNRAS, 483, 172

Duckworth C., Tojeiro R., Kraljic K., 2020, MNRAS, 492, 1869

Emsellem E., et al., 2007, MNRAS, 379, 401

Falcón-Barroso J., Sánchez-Blázquez P., Vazdekis A., Ricciardelli E., Cardiel N., Cenarro A. J., Gorgas J., Peletier R. F., 2011, A\&A, 532, A95

Ganeshaiah Veena P., Cautum M., van de Weygaert R., Tempel E., Jones B. J. T., Rieder S., Frenk C. S., 2018, MNRAS, 481, 414

Ganeshaiah Veena P., Cautun M., Tempel E., van de Weygaert R., Frenk C. S., 2019, arXiv e-prints,

Gunn J. E., et al., 2006, AJ, 131, 2332

Hahn O., Carollo C. M., Porciani C., Dekel A., 2007, MNRAS, 381, 41

Haynes M. P,, Giovanelli R., 1984, AJ, 89, 758

Hiry A., Pelt J., Saar E., Tago E., Tamm A., Tempel E., Einasto M., 2017, A\&A, 599, A31

Hoyle F., 1949, Problems of Cosmical Aerodynamics, Central Air Documents, Office, Dayton, OH. Central Air Documents Office, Dayton, $\mathrm{OH}$

Joachimi B., Mandelbaum R., Abdalla F. B., Bridle S. L., 2011, A\&A, 527, A26

Johnston H., et al., 2019, A\&A, 624, A30

Jones B. J. T., van de Weygaert R., Aragón-Calvo M. A., 2010, MNRAS, 408, 897

Krajnović D., Cappellari M., de Zeeuw P. T., Copin Y., 2006, MNRAS, 366, 787

Kraljic K., et al., 2020a, MNRAS, 491, 4294

Kraljic K., Davé R., Pichon C., 2020b, MNRAS, 493, 362

Krolewski A., Ho S., Chen Y.-C., Chan P. F., Tenneti A., Bizyaev D., Kraljic K., 2019, arXiv e-prints,

Law D. R., et al., 2015, AJ, 150, 19

Law D. R., et al., 2016, AJ, 152, 83

Lee J., 2011, ApJ, 732, 99

Lee J., Erdogdu P., 2007, ApJ, 671, 1248

Lee J., Libeskind N. I., 2020, arXiv e-prints, p. arXiv:2008.02121

Lee J., Pen U.-L., 2000, ApJ, 532, L5

Lee J., Pen U.-L., 2001, ApJ, 555, 106

Lee J., Libeskind N. I., Ryu S., 2020, ApJ, 898, L27

Nair P. B., Abraham R. G., 2010, ApJS, 186, 427

Okumura T., Jing Y. P., 2009, ApJ, 694, L83

Pahwa I., et al., 2016, MNRAS, 457, 695

Peebles P. J. E., 1969, ApJ, 155, 393

Sánchez-Blázquez P., et al., 2006, MNRAS, 371, 703

Schäfer B. M., 2009, International Journal of Modern Physics D, 18, 173

Singh S., Mandelbaum R., More S., 2015, MNRAS, 450, 2195 
Smee S. A., et al., 2013, AJ, 146, 32

Sousbie T., 2011, MNRAS, 414, 350

Sousbie T., Pichon C., Kawahara H., 2011, MNRAS, 414, 384

Tempel E., Libeskind N. I., 2013, ApJ, 775

Tempel E., Stoica R. S., Saar E., 2013, MNRAS, 428, 1827

Tempel E., et al., 2014, A\&A, 566, A1

Trowland H. E., Lewis G. F., Bland-Hawthorn J., 2013, ApJ, 762, 72

Wake D. A., et al., 2017, AJ, 154, 86

Wang P., Kang X., 2017, MNRAS, 468, L123

Wang P., Guo Q., Kang X., Libeskind N. I., 2018, ApJ, 866, 138

Welker C., et al., 2020, MNRAS, 491, 2864

Westfall K. B., et al., 2019, arXiv e-prints, p. arXiv:1901.00856

White S. D. M., 1984, ApJ, 286, 38

Willett K. W., et al., 2013, MNRAS, 435, 2835

Yan R., et al., 2016a, AJ, 151, 8

Yan R., et al., 2016b, AJ, 152, 197

Zhang Y., Yang X., Wang H., Wang L., Mo H. J., van den Bosch F. C., 2013, ApJ, 779, 160

Zhang Y., Yang X., Wang H., Wang L., Luo W., Mo H. J., van den Bosch F. C., 2015, ApJ, 798, 17

\section{APPENDIX A: TWO-FOLD AMBIGUITY OF ROTATION}

Figures A1 and A2 show the measured alignment signal when statistically accounting for the clock-wise and counter-clockwise rotation, as proposed by Lee (2011). This consists in assigning to each galaxy a set of two unit spin vectors differing from each other by the sign of $\hat{L}_{r}$, i.e. in addition to the spin vector defined by Eq.3, we assign to each galaxy spin vector defined as

$\hat{L}_{x}^{-}=-\hat{L}_{r} \sin \alpha \cos \beta+\hat{L}_{\theta} \cos \alpha \cos \beta-\hat{L}_{\phi} \sin \beta$

$\hat{L}_{y}^{-}=-\hat{L}_{r} \sin \alpha \sin \beta+\hat{L}_{\theta} \cos \alpha \sin \beta+\hat{L}_{\phi} \cos \beta$

$\hat{L}_{z}^{-}=-\hat{L}_{r} \cos \alpha-\hat{L}_{\theta} \sin \alpha$.

LTGs (Fig. A1) tend to have their spin parallel to their host closest filaments, while S0s (Fig. A2) tend to have their spin in the orthogonal direction.

This paper has been typeset from a $\mathrm{T}_{\mathrm{E}} \mathrm{X} / \mathrm{L} \mathrm{T} \mathrm{E} \mathrm{X}$ file prepared by the author.

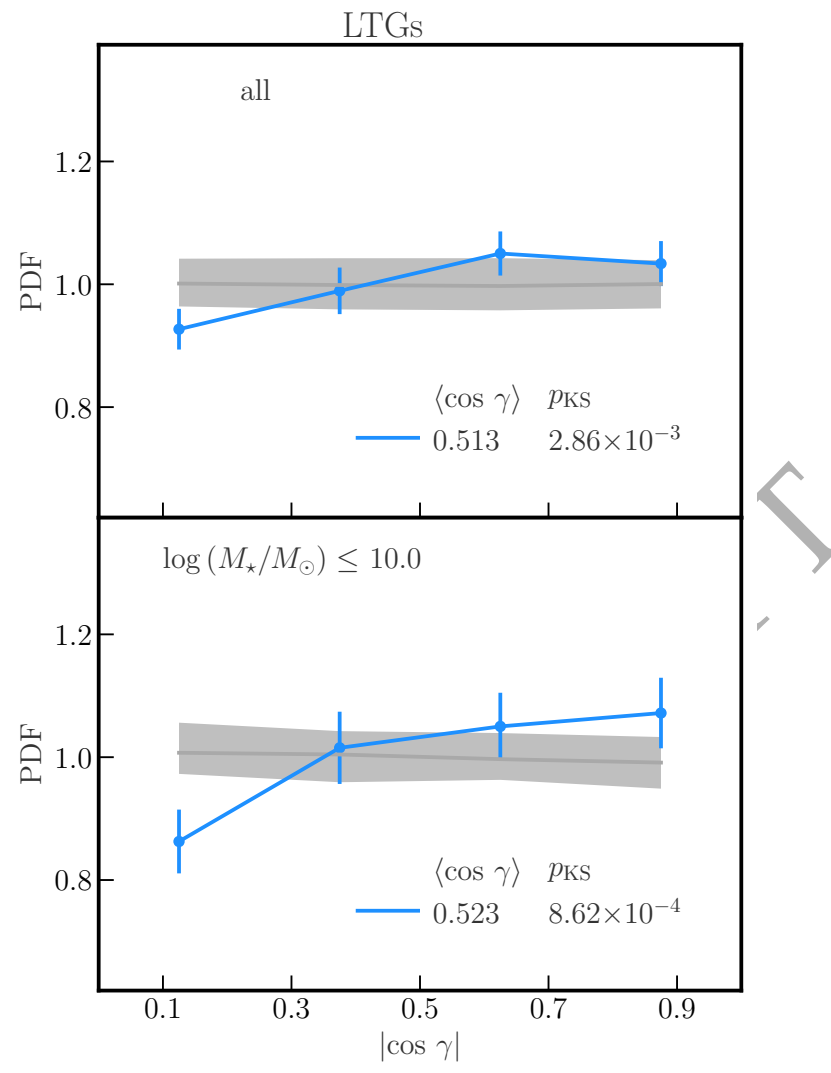

Figure A1. Alignment between the filaments and spin of all (top), and, lowmass (bottom) LTGs. The error bars correspond to the bootstrap. The solid grey line and shaded area represent the median and 95 per cent confidence limits from 5000 random samples, respectively. The mean alignment angle together with the probability $p_{\mathrm{KS}}$ of the KS test are shown in each panel with corresponding symbols. LTGs in the probed mass range tend to have their spin parallel to their host filaments. The alignment signal seen for the entire population of LTGs is driven by the low mass sub-sample $\left(M_{\star} \leq\right.$ $\left.10^{10} \mathrm{M}_{\odot}\right)$. 


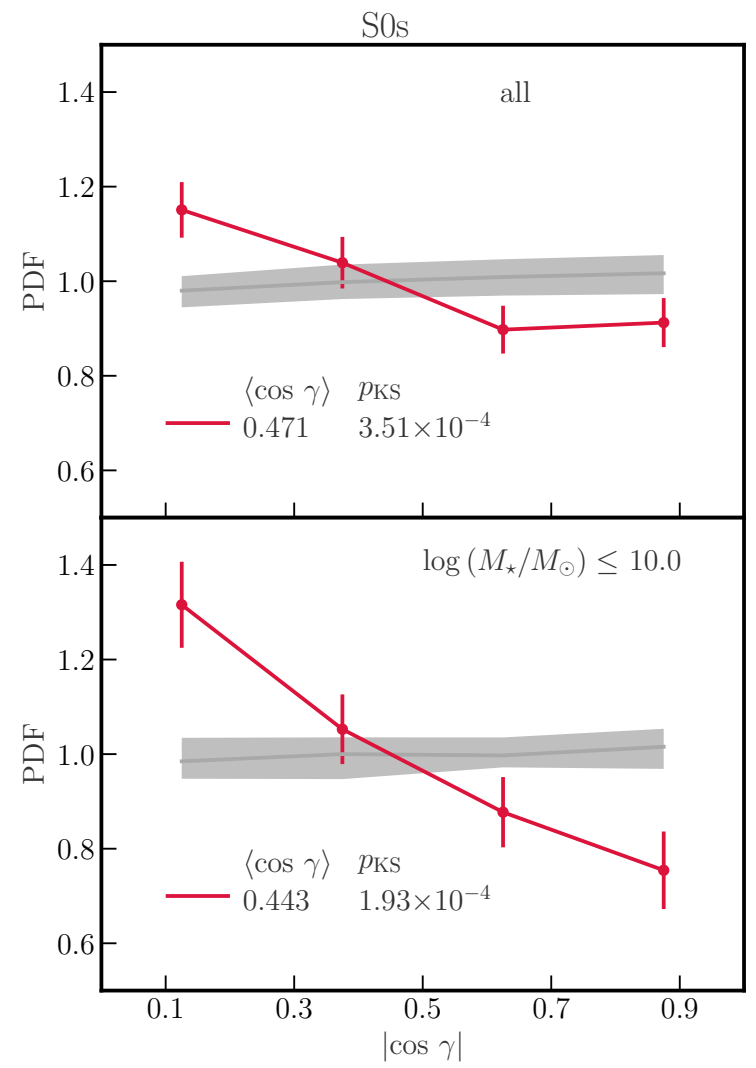

Figure A2. Alignment between the filaments and spin of all (top) and lowmass (bottom) S0s. The error bars correspond to the bootstrap. The solid grey line and shaded area represent the median and 95 per cent confidence limits from 5000 random samples, respectively. The mean alignment angle together with the probability $p_{\mathrm{KS}}$ of the KS test are shown in each panel with corresponding symbols. S0s in the probed mass range tend to have their spin perpendicular to their host filaments. The alignment signal seen for the entire population of S0s is driven by the low mass sub-sample ( $M_{\star}$ $\left.\leq 10^{10} \mathrm{M}_{\odot}\right)$. 